\title{
Predictive Safety Management System Development
}

\section{Dajana Bartulović}

Safety management systems are used to systematically manage safety risks. The paper describes and explains safety management systems in the field of aviation. Three aviation safety management methodologies are presented in the paper: reactive, proactive, and predictive. The aim is to show how safety management systems operate in each of the three methodologies. The focus of the paper is on predictive safety management methodology, its advantages, and potential uses. An overview of predictive methods used in the aviation industry is also provided. The research collected information on each safety management methodology, and revealed correlations between them, improving our understanding of safety management systems in general. Based on research described in the paper, the author proposes the development of a more advanced safety management system, i.e. a predictive safety management system which would entail the development of an expanded and well-organised safety database, as well as the use of predictive (forecasting) methods to identify potential and emerging hazards, trends and behaviour patterns.

\section{KEY WORDS}

$\sim$ Development

$\sim$ Predictive

$\sim$ Methodology

$\sim$ Aviation

$\sim$ Safety management system

Institute of Traffic and Communications, Zagreb, Croatia

e-mail:dbartulovic@gmail.com

doi: 10.7225/toms.v10.n01.010

This work is licensed under (cc) BY

Received on: Sep 9, 2020 / Revised on: Feb 12, 2021 / Accepted on: Feb 14, 2021 / Published: Apr 20, 2021

\section{INTRODUCTION}

Since their introduction in the field of aviation, safety management systems have greatly contributed to aviation safety. Nowadays, every aviation organisation is obligated to implement a safety management system and actively record any event (hazard) that has occurred or could potentially occur in the organisation. Aviation system growth requires the introduction of advanced safety capabilities that increase capacity while maintaining or enhancing operational safety and managing existing and emerging risks more efficiently.

The paper explains three main methodologies used in aviation safety management: reactive, proactive, and predictive. It outlines benefits and capabilities of each methodology. The research revealed correlations between them, improving our understanding of safety management systems in general.

The aim of this paper is to present predictive methodology of safety management, i.e. its advantages, current application in the aviation industry and possible future development.

An overview of predictive methods used in aviation industry is given in this paper. Based on scientific research described in this paper, that involved the application of different scientific methods, such as the inductive and deductive method, the analysis and synthesis method, the generalization and specialization method, the classification method, the descriptive method, the compilation method, and the comparative method; the author proposes the development of a more advanced safety management system, i.e. a predictive safety management system which would entail the development of an expanded and well-organised safety database, as well as the use of predictive (forecasting) methods to identify potential and emerging hazards, trends and behaviour patterns.

Outlining the concept of predictive methodology implementation and development as an additional safety management system tool is the scientific contribution of this 
paper. Using data analysis and predictive methods to identify potential and emerging hazards can help prevent future adverse events in the operations of any organization.

\section{DEFINITION AND OVERVIEW OF AVIATION SAFETY MANAGEMENT SYSTEM DEVELOPMENT}

A Safety Management System (SMS) is a formal organizational system that manages safety. It integrates active safety management tools, including senior management commitment, hazard identification, risk management, risk mitigation, safety reporting, audit, investigations and remedial actions, safety culture and education supported by clear policies and processes. (ICAO SMM Doc 9859, 2018; Bangladesh Civil Aviation Authority, 2010)

The traditional approach focused on compliance with increasingly complex regulatory requirements, which functioned well until the late 1970s, when the trend came to a stagnation point in the number of accidents and incidents. Accidents continued to happen despite the continuous improvement of rules and regulations. This approach to safety was reactive, as it reacted to events that had already occurred by adopting regulations aimed at preventing their recurrence. (BHDCA, 2014; Stolzer and Goglia, 2015; Steiner, 1998)

The modern approach is shifting away from reactive towards a proactive approach. In addition to existing rules and regulations, a number of other activities that improve flight safety need to be developed: the adoption of risk assessment methods, the commitment of administrative bodies in flight safety management, the development of an organizational culture that encourages safe practices and communication and actively manages flight safety, effective implementation of standard operating procedures, including the use of checklists and briefings, a "just culture" environment that encourages effective hazard and incident reporting, the establishment of a system for the collection, analysis and exchange of significant safety data obtained in the course of normal operations, the investigation of accidents and serious incidents aimed at identifying systemic shortcomings rather than finding the culprit, introduction of flight safety training (including the human factor) as a requirement for all operational staff, the exchange of know-how and best practices through active exchange of safety information (between organizations and states), systematic safety monitoring and performance monitoring to evaluate system condition to reduce or eliminate problem areas.

In the modern system, the greatest attention is paid to building a positive organizational culture, which frequently involves overcoming the negative aspects of existing national and professional cultures. (BHDCA, 2014)

The SMS strategy adopted by an organization will reflect its corporate safety culture that can vary from purely reactive, responding only to the occurrence of accidents, to strategies that are highly proactive in their search for security issues. Traditional or reactive, the process is characterized by subsequent repairs. In a modern or proactive approach, preventive reform plays a major role.

According to (Adjekum, 2014), Safety Management System (SMS) is also an organized approach to systemic safety improvement. Safety management, as an organizational process and a core business function, clearly places ultimate accountability and responsibility for safety on the highest level of any aviation organization.

Safety Management Systems (SMS) are a mechanism that is used to improve an industry which already has an exceptional aviation safety record. The ICAO defines SMS as an organized approach to safety management, that includes necessary organizational structures, accountabilities, policies, and procedures. The four pillars of SMS are: safety policy and objectives, risk management, safety assurance, and safety promotion. According to (ICAO SMM Doc 9859, 2018; Croatian Civil Aviation Agency, 2016; Velazquez and Bier, 2015; Ferguson and Nelson, 2014; Cusick et al., 2017; State Safety Programme, 2015) the framework of organisational SMS should include the four previously mentioned components and the accompanying 12 elements: 1 . safety policy and objectives: 1.1 management commitment ,1.2 safety accountability and responsibilities, 1.3 appointment of key safety personnel, 1.4 coordination of emergency response planning, 1.5 SMS documentation; 2. safety risk management: 2.1 hazard identification, 2.2 safety risk assessment and mitigation; 3 . safety assurance: 3.1 safety performance monitoring and measurement, 3.2 the management of change, 3.3 continuous improvement of the SMS; 4. safety promotion: 4.1 training and education, 4.2 safety communication. Error, hazard and risk management and control are all part of the safety system defined as SMS.

It is important to recognise that SMS is a top down driven system, which means that the accountable manager of an organisation is responsible for the implementation and continuous compliance with the SMS. Without the full support of the accountable manager, SMS will not be effective. There is no 'one size fits all' model of SMS that would cater to all types and sizes of service providers. Complex SMS systems are likely to be inappropriate for small organisations. Therefore, such organisations should tailor their SMS to suit their size, nature and complexity of their activities and allocate resources accordingly. (ICAO SMM Doc 9859, 2018; Croatian Civil Aviation Agency, 2016)

Safety management systems are commonly used in the aviation domain to systematically manage risks to aviation safety. ICAO-based SMS principles (ICAO SMM Doc 9859, 2018; Australian Government Defence Aviation Safety Authority, 2015) allow for the following: SMSs to be tailored to the scope of equipment/ aircraft, operations and maintenance to be conducted by the 
unit; a phased SMS introduction based on complementary organisational culture change management programs and timelines; and agreement of aviation regulators about the scope of individual SMS plans.

However, as indicated in the Civil Aviation Authority of New Zealand and Burin (Civil Aviation Authority of New Zealand, 2013; Burin, 2013), modern data collection and analysis capabilities allow us to make predictions that enable us to take a closer look at the previously identified high-risk areas, to gain a better insight into the (in)effectiveness of risk-reduction efforts and potentially identify risk-reduction gaps that have previously eluded us.

\section{OVERVIEW OF AVIATION SAFETY MANAGEMENT SYSTEM METHODOLOGIES}

Safety management systems have greatly contributed to aviation safety since their introduction in this field. Today, every aviation organisation is under obligation to implement a safety management system (SMS) and actively record and report every event (hazard) that has occurred or could potentially occur in the organisation. To ensure that continuous safety improvement and global air navigation modernization advance hand-in-hand, global, regional and national aviation safety planning is essential.

The ICAO's Global Plans define the means and targets by which the ICAO, countries and aviation stakeholders can anticipate and efficiently manage air traffic growth, while proactively maintaining or improving safety. Policies, procedures and systems that allow civil aviation to realize the above goals, while remaining safe, secure, efficient, and environmentally sustainable, are prescribed by ICAO's coordinated international Standards and Recommended Practices (SARPs). All of these activities are harmonized in keeping with the principles and objectives outlined in ICAO's Global Aviation Safety Plan (GASP), as well as in Annex 19 on Safety Management (ICAO Annex 19, 2016; ICAO SGAS, 2013)

On the global level, the International Civil Aviation Organisation (ICAO) prescribes 19 Annexes of Standard and Recommended Practices (SARPs), among which Annex 19 (ICAO Annex 19, 2016) contains rules and regulations on Safety Management and the ICAO Safety Management Manual (ICAO SMM Doc 9859, 2018), as a guide helping each member state implement State Safety Programmes at the national level and Safety Management Systems at the aviation organisation level. In the European Union (EU), the authority delegated the power to prescribe rules is the European Union Aviation Safety Agency (EASA). The EASA brings safety reporting and accident investigation regulations, as well as general regulations on the implementation of safety management systems by organisations on EU territory. The Commission Regulation (EU) 376/2014 (Commission Regulation (EU) 376/2014, 2014) and Implementing Regulation (EU) 2015/1018 (Implementing Regulation (EU) 2015/1018, 2015) regulate safety reporting and reportable

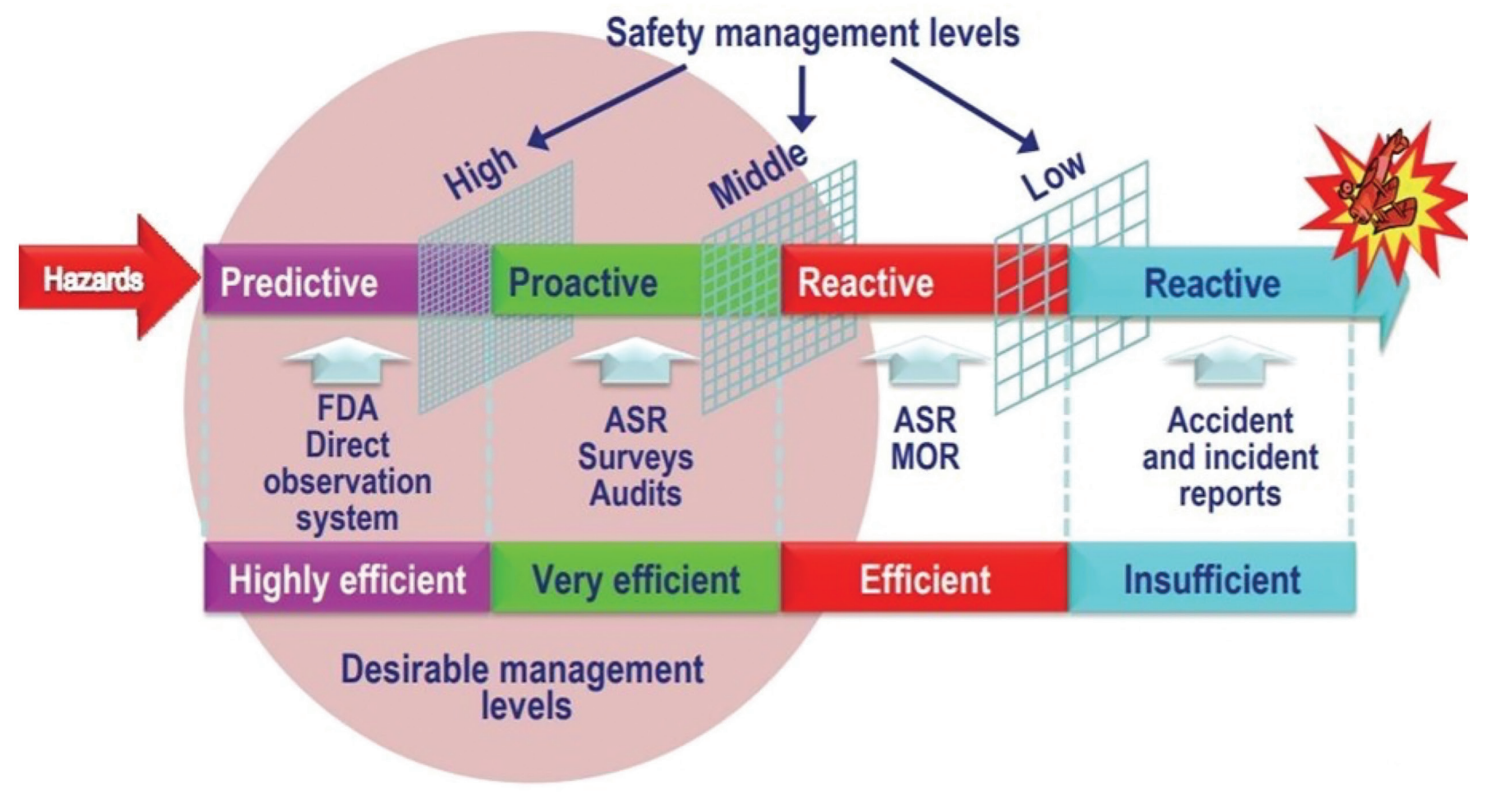

Figure 1.

Safety management levels.

Source: ICAO, 2011 
occurrences, while Commission Regulation (EU) 965/2012: PartORO (Commission Regulation (EU) 965/2012, 2012), Commission Regulation (EU) 1178/2011: Part-ORA (Commission Regulation (EU) 1178/2011, 2011), Commission Regulation (EU) 1321/2014 (Commission Regulation (EU) 1321/2014, 2014) and Commission Regulation (EU) 139/2014: Part-ADR.OR (Commission Regulation (EU) 139/2014, 2014), and other, regulate the implementation and maintenance of effective SMS by every operator or organisation providing services in the field of aviation.

The implementation and maintenance of an effective SMS require each aviation organisation to comply with all regulations mentioned above. Effective SMS has to have four main components in place to work properly and efficiently. Those four components, as previously mentioned, are safety policy, safety risk management, safety assurance and safety promotion. The second component, Safety Risk Management (SRM), is at the core of efficient SMS. It deals with occurrence (hazard) identification, risk assessment and risk mitigation. (Čokorilo and Dell'Acqua, 2013; Čokorilo et al., 2011; Jakovljević et al., 2017; Steiner, 1998; Bartulović, 2012) The third component, Safety Assurance, includes safety performance monitoring and measurement, change management and continuous improvement of the SMS.

The SMS also defines three management methodologies: reactive, proactive, and predictive (Figure 1). (Oster Jr. et al., 2013; ICAO SMM Doc 9859, 2018)

All three methodologies are closely linked to all four above mentioned components, especially the safety risk management component, in particular hazard identification. The SMS needs input data to identify hazards, i.e. to be able to provide viable results and these methodologies are the tool that enables SMS to acquire the necessary safety data.

Three categories of reports are collected: mandatory, voluntary and changes (Ordinance on the implementation of Regulation (EU) 376/2014, 2015). Mandatory reports pertain to the set of occurrences required to be reported on by applicable regulations. Voluntary reports record potentially hazardous occurrences falling outside the scope of mandatory occurrences. Reports on changes record every change that happens inside or outside an organisation, since every change is potentially hazardous. These reports may refer to internal (within an organisation) or external changes (usually in regulations).

\subsection{Reactive Safety Management System}

Reactive methodology collects safety data from accidents and incidents that have already occurred and learns from their outcomes. As mandatory reports are drawn up after the occurrence, mandatory occurrence reporting can be classified as a reactive safety data collection methodology.

According to Bangladesh Civil Aviation Authority (Bangladesh Civil Aviation Authority, 2010), "reactive navigation aids" require a very serious trigger, often with considerable negative consequences, to launch the safety data capture process. The contribution of reactive navigation aids to safety management nevertheless depends on the extent to which the information they generate go beyond identifying the cause of the event, and allocating blame, and include contributory factors and findings relevant for safety risks. (Bohm, 2008) Accident and serious incident investigations are examples of reactive navigation aids. Other examples are situations involving technology failures, or unusual events.

According to Cusick and Airbus Safety Magazine (Cusick et al., 2017; Airbus Safety Magazine: Safety First, 2014), reactive methodology can be defined as follows: hazards are identified through investigation and analysis of past incidents or accidents, i.e. safety occurrences. Incidents and accidents are potential indicators of systems' deficiencies and can therefore be used to determine the hazards that contributed to the event or are latent.

An illustration of reactive a safety management system and its most important activities is shown in Figure 2.
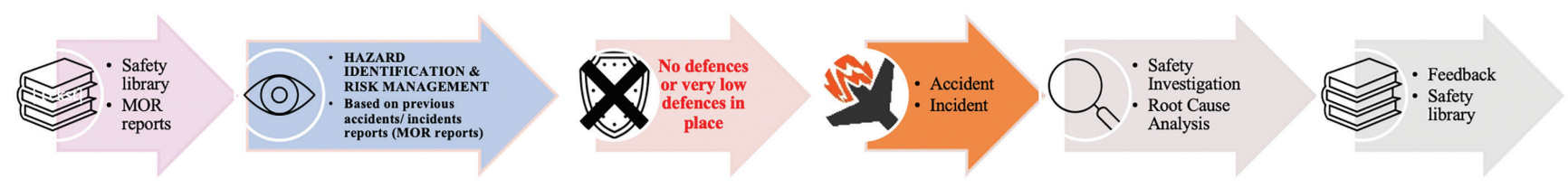

Figure 2.

Reactive safety management system.

Source: Author 


\subsection{Proactive Safety Management System}

Proactive methodology uses safety reporting systems and safety performance indicators to gather safety data in order to discover and mitigate the potential threats and hazards that could trigger accidents or incidents.

Voluntary reports and change reports record potential threats and hazards capable of triggering more serious occurrences; such reports are therefore classified as proactive methodology of safety management.

Proactive methodology collects safety data on occurrences or an organisation's process performance and analyses the collected safety data or their frequency to estimate a hazard's potential to trigger an accident or an incident.

The main safety data collection mechanism used in proactive methodology is the safety reporting system. Safety data can be collected from various types of safety reports such as: accident or incident investigations, voluntary safety reporting system, change management, continuing airworthiness reports, operational performance monitoring (flight data analyses), inspections, audits, surveys or safety studies and reviews.

The main activities of proactive safety management methodology include defining Safety Performance Indicators (SPIs) and setting Safety Performance Targets (SPTs) (ICAO SMM Doc 9859, 2018).
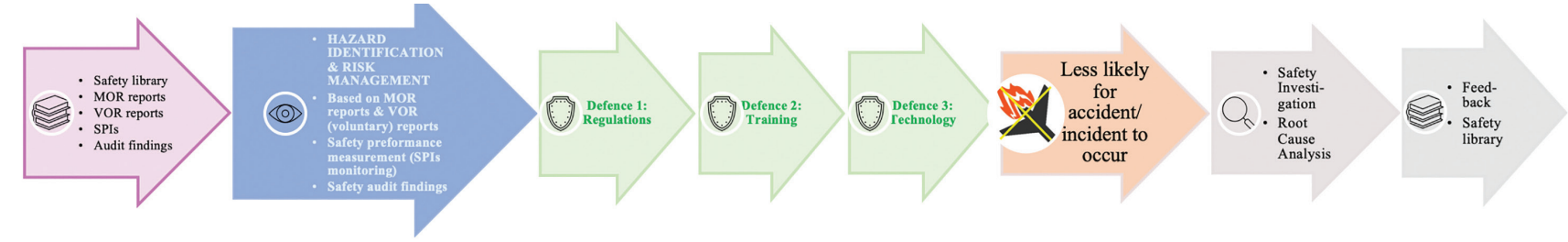
now; and where it is headed in terms of its safety SPIs should therefore be realistic, relevant, and linked to safety objectives of the organisation. Safety performance targets (SPTs) define the desired safety performances. They ensure that the organization is on track to achieving its safety objectives and provide a measurable way of verifying the effectiveness of safety performance management activities. SPIs and SPTs give a clear picture of an organization's safety performance.

According to Bohm and Cusick (Bohm, 2008; Cusick et al., 2017), proactive safety management: identifies safety risks within the system before it fails; and takes the necessary actions to reduce such safety risks.

According to Airbus Safety Magazine (Airbus Safety Magazine: Safety First, 2014), definition of proactive methodology is: hazards are identified through an analysis of an organisation's activities before hazards materialize into incidents or accidents and the necessary actions are taken to reduce the associated safety risks. The proactive process is based on the notion that safety events can be minimized by identifying safety risks within the system before it fails, and taking the necessary actions to mitigate such safety risks.

An illustration of proactive safety management system and its most important activities is given in Figure 3.

Figure 3.

Proactive safety management system.

Source: Author

\subsection{Predictive Safety Management System}

Predictive methodology is as yet not well established, as it aims to identify potential and possible hazards based on predictive analyses (forecasts) that extract information from historical and current safety data and to predict trends and behaviour patterns of emerging hazards (Ancel et al., 2015, Čokorilo et al., 2019; ICAO SMM Doc 9859, 2018; Luxhoj, 2013; Stanton et al., 2008; Cusick et al., 2017).

Predictive SMS methodology can use historical and current safety data, SPIs and SPTs of an organisation (Bartulović and Steiner, 2020) as input information to conduct predictive analysis, i.e. make forecasts using predictive (forecasting) methods. The obtained results show trends and behaviour patterns of established SPIs in the organisation and give a clearer view of the future development of an organization's safety performance, while simultaneously identifying emerging hazards.

As stated by Airbus Safety Magazine and the Bangladesh Civil Aviation Authority (Airbus Safety Magazine: Safety First, 2014; Bangladesh Civil Aviation Authority, 2010), predictive navigation aids do not require the occurrence of a triggering event to launch the safety data capture process. Routine operational data are continuously collected in real time. Predictive navigation aids are based on the notion that safety management is best 


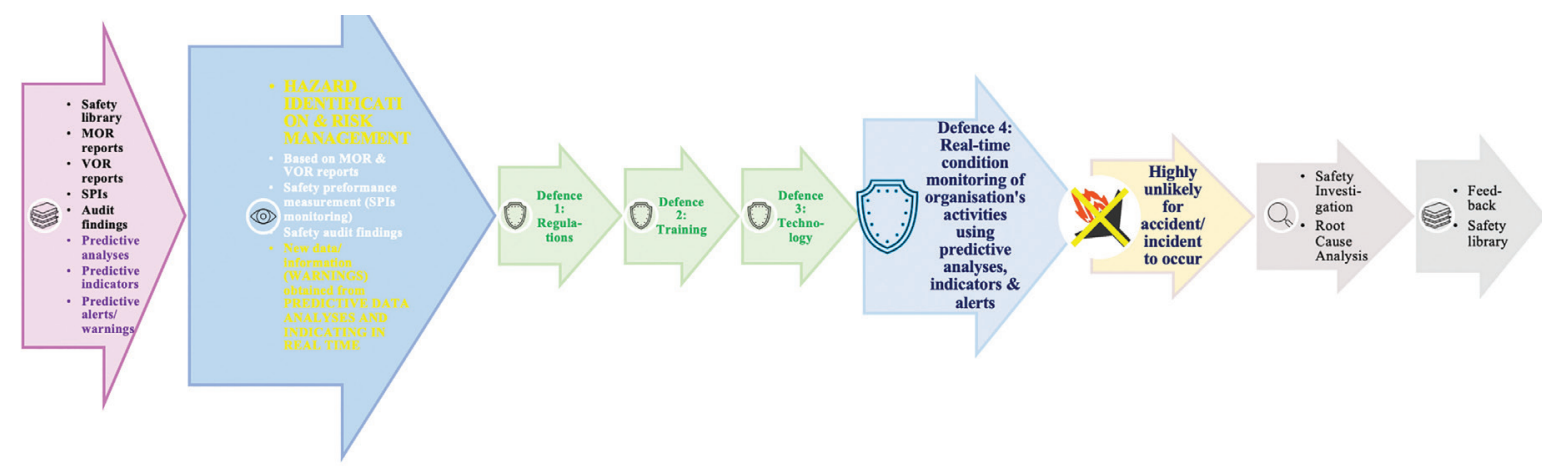

Figure 4.

Predictive safety management system.

Source: Author

accomplished by trying to identify a problem instead of simply waiting for something to happen. Therefore, predictive safety data capture systems aggressively seek safety information that could be indicative of emerging safety risks from a variety of sources.

An illustration of predictive safety management system and its most important activities is given in Figure 4.

\section{OVERVIEW OF PREDICTIVE METHODS USED IN AVIATION INDUSTRY}

This chapter gives an overview of predictive methods used in the aviation industry to improve some aspects of safety.

Some predictive methods that can be used to analyse safety data are, for example linear trend analysis and moving average (Brockwell and Davis, 2016; Bartulović and Steiner, 2020). Safety data can be an organization's historical safety data that can be used to predict future behaviour of monitored parameters or indicators.

AFCAC (AFCAC, 2018) suggests the implementation of predictive safety systems. It emphasizes the fact that safety system integration is possible through the use of an appropriate modular software suite which should encompass all of the major safety oversight responsibilities and typical automated systems that are already used by a considerable number of regulators worldwide. The software architecture focus areas should include: legislation; organisation; SSP and SMS; personnel licensing (examinations and licensing); flight operations; aircraft Incidents/ accidents; airworthiness; aerodromes; air navigation services. Safety records and data should be stored in a single, fully crossreferenced database (for each regulator) that should allow detailed analysis of safety risks pertinent for discrete areas of oversight, as well as across the entire aviation industry. Safety data and safety information management that address the necessary functions should ensure that an organization's safety data and safety information are collected, stored, analysed, retained and archived, as well as governed, protected and shared.

Boeing (Boeing, 2012) develops and incorporates new technologies to enhance safety. Through research, development and collaboration, Boeing has developed sophisticated technologies that provide distinct safety advantages, such as: Vertical Situation Display, predictive windshear equipment, as well as improved windshear - training programs for pilots, and the Enhanced Ground Proximity Warning System.

The ICAO (ICAO GASP, 2013) states that the focus of longterm objectives is the implementation of predictive systems that will become an integral part of the aviation systems of the future. Sustainable growth of the international aviation system will require the introduction of advanced safety capabilities that increase capacity while maintaining or enhancing operational safety margins and managing existing and emerging risks. The long-term objective is to support an operational environment characterized by increased automation and integrate advanced capabilities on the ground and in the air. The ICAO is committed to the development and implementation of new safety initiatives in response to concerning trends in safety data. Aviation systems will become increasing automated and infinitely more complex, which could potentially change the role of aviation professionals. Safety oversights under such circumstances will require the use of proactive and predictive risk modelling capabilities. This approach will allow the aviation community to effectively monitor the health of the aviation system, virtually in realtime, and make necessary adjustments to maintain the desired safety levels. The ICAO (ICAO GASP, 2013) has started granting significantly improved and greater online access to real-time safety information through its iSTARS (Integrated Safety Trend Analysis and Reporting System) initiative, as well as access to a range of additional aviation data, to support the implementation 
of the evolving approach to safety management. A series of objectives support this aspirational safety goal. The ICAO 20202022 edition of the GASP (ICAO GASP, 2019) calls on the States to implement effective safety oversight systems, and SSPs, and move towards predictive risk management.

The ITF (ITF, 2018) points out that growing complexity of the transportation system has allowed the industry to carry an ever-increasing number of passengers and freight volumes, at an ever-decreasing real cost. Growing complexity has also introduced new hazards into the transportation system and thus requires proper predictive risk analysis and mitigation that should be made a part of the SMS.

Khoshkhoo (Khoshkhoo, 2017) developed Dispatch Operations Safety Audit (DOSA) - a proactive and predictive safety management system method that identifies the advantages and drawbacks of dispatcher performance. Potential applications of this research include improved threat and error management in the Operations Control Centre (OCC), as well as the identification of threats and error types.

Liou conducted (Liou, 2008) a research to better understand the role of the human factor in major aviation accidents. A method for building an effective safety management system for airlines was developed that incorporates organization and management factors. It combines both fuzzy logic and DecisionMaking Trial and Evaluation Laboratory (DEMATEL). This method can map out the structural relations among diverse factors in a complex system and identify key factors.
In 2011, Panagopoulos (Panagopoulos, 2011) researched military pilot error framework. The intent was to start to bridge and compare the existent, mostly reactive, Flight Safety programmes implemented in NATO/EU Air Forces and show how a more proactive and predictive Safety Management System can be realised.

Pisanich and Corker (Pisanich and Corker, 1995) described Air-MIDAS, a model of pilot performance in interaction with varied levels of automation inflight management operations. The model was used to predict the performance of a two-person flight crew responding to clearance information generated by the Centre TRACON Automation System (CTAS). The model incorporates information requirements, decision processes, communication processes, and motor performances required by the flight crew to integrate flight management automation and ground-side automation into clearance assistance. The paper described the model, its development and implementation, the model prediction simulation test, and the empirical validation process. The complex human performance model allows variations in CTAS design to be explored through predictive simulation. Procedures and performance criteria, as well as situational variations, can be controlled and tested. The model and its supporting data provide a generalizable tool that can be expanded to include air/ground compatibility and ATC crew interactions in air traffic management.

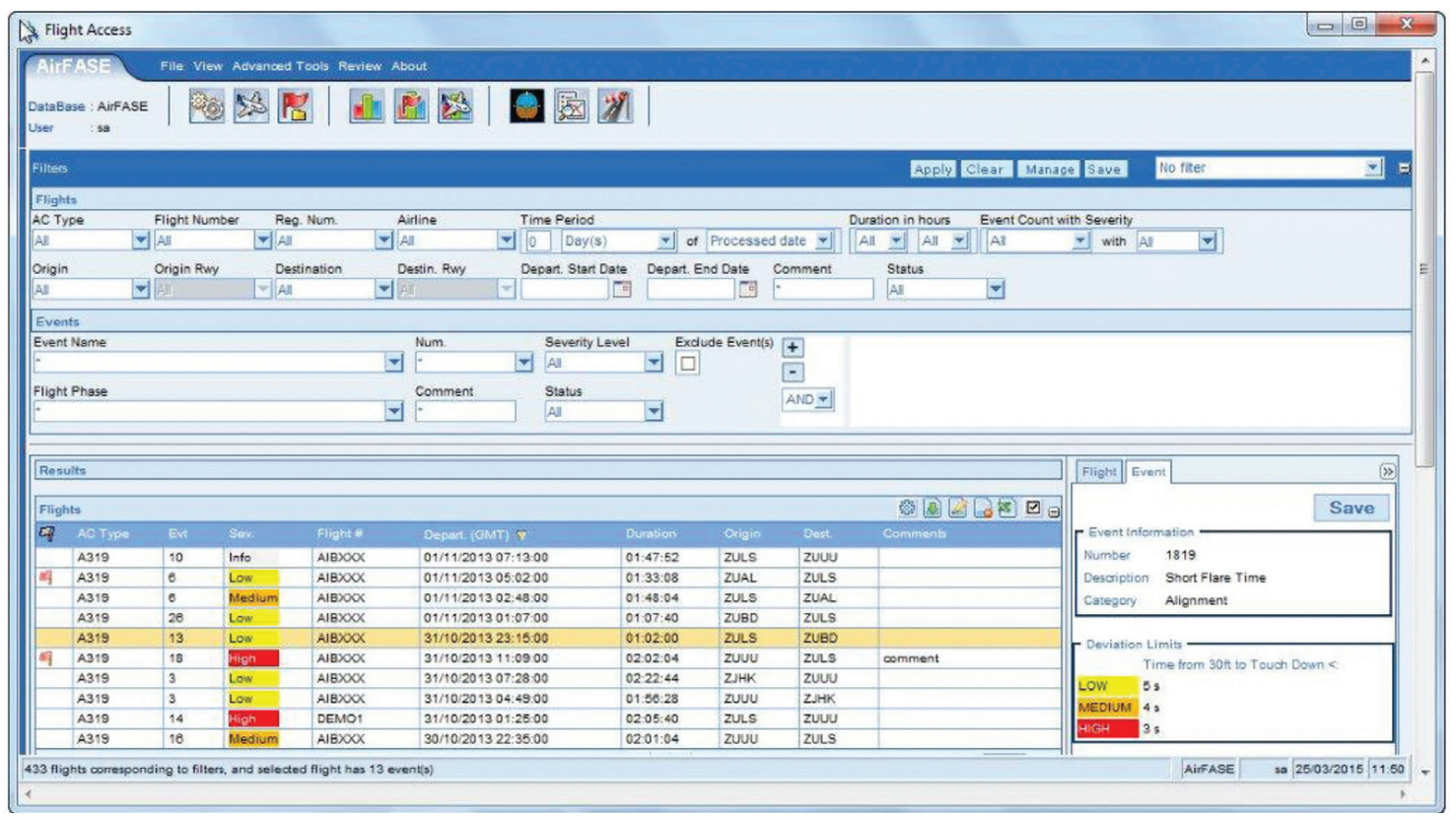

Figure 5.

Example of the Flight Data Analysis Suite.

Source: NavBlue, 2020 
Roelen and others (Roelen et al., 2016) studied the integrated approach to risk modelling that takes into account the overall aviation system, where the human factor and cultural aspects are considered in connection with technical and procedural aspects, with an emphasis on the representation of emerging and future risks. Specific objectives were to: test the safety of the complete current aviation system in accident scenarios; study emerging and future risks in accident scenarios; explore safety culture and safety management in accident scenarios; and explain how accident scenarios can be quantified.

As stated in the Airbus Safety Magazine (Airbus Safety Magazine: Safety First, 2014), recording technology has significantly improved - from analogue to digital on tape, then to solid state able to record over 3,000 parameters. In the meantime, Flight Data Monitoring processes were encouraged and sometimes even requested by authorities. Today, while Flight Data Recorders (FDRs) or Digital Flight Data Recorders (DFDRs) are dedicated to accident investigation, Flight Data Analysis programs (Figure 5) extract data from easily accessible recorders and customize the recorded parameters. FDR logically led to FDA and the reactive process evolved into a predictive process. Analysts manually filter the data. They look for all high deviation magnitude events in order to assess any serious safety concern and take appropriate corrective action. Correlating them with any other means, like mandatory or voluntary reports, will multiply analysis efficiency. All reliable events are stored in a database and investigated on a regular basis to highlight any trend that could constitute a latent or potential risk.

\section{CORRELATION BETWEEN REACTIVE, PROACTIVE AND PREDICTIVE SAFETY MANAGEMENT METHODOLOGIES}

Chapter 2 defines and explains safety management systems and their origins. Chapter 3 gives an overview and describes three safety management methodologies: reactive, proactive, and

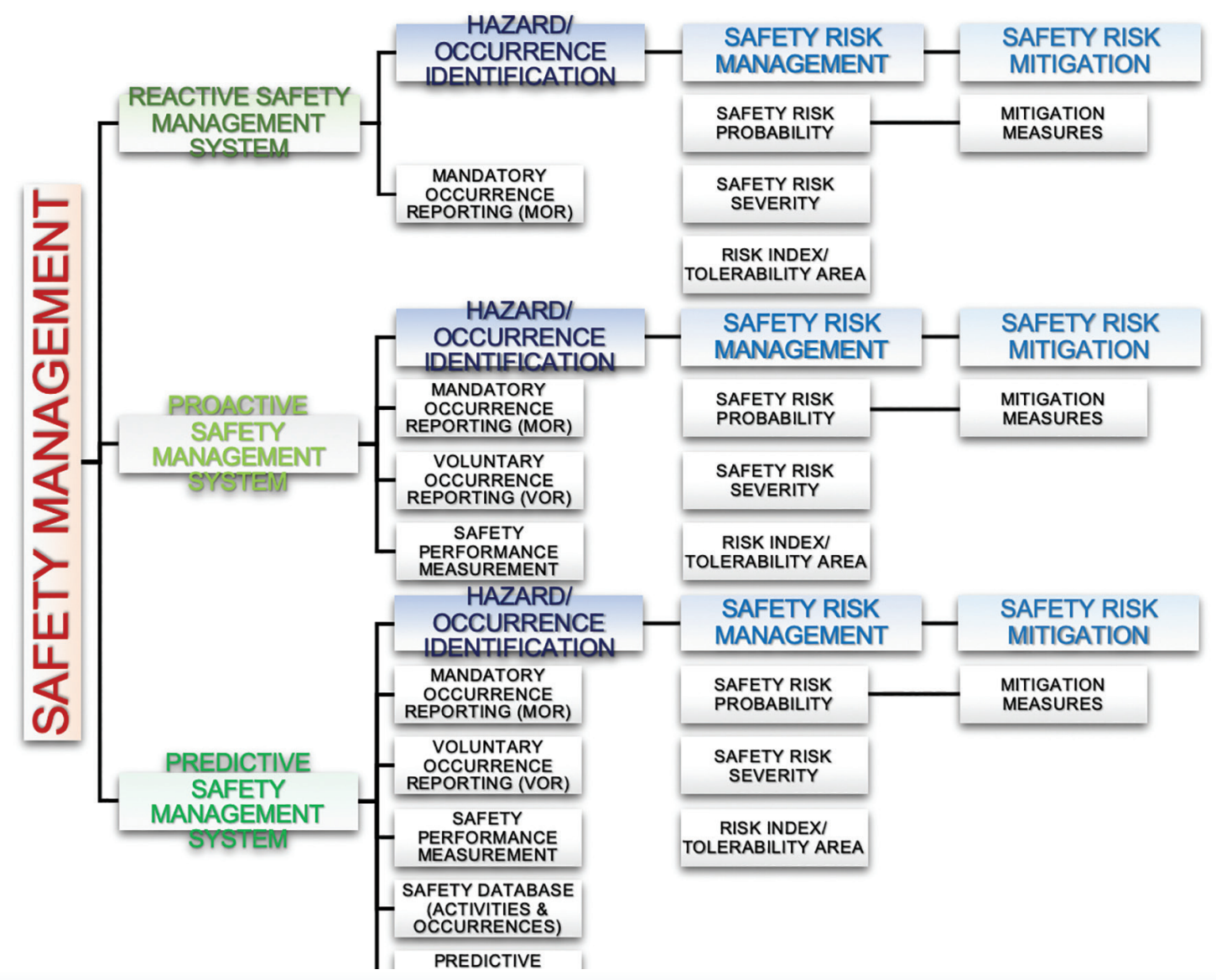

Figure 6.

Overview of safety management system methodologies.

Source: Author 
predictive. The examination of all three methodologies revealed both their mutual differences and, more importantly, similarities. Each of the three methodologies is a specific approach to safety management, i.e. depending on the development of the safety management system in a specific organisation, it can adopt a reactive, proactive, or predictive safety management system. Each approach resolves safety issues by following the same key steps: hazard identification, safety risk assessment and safety risk mitigation (Figure 6).

However, safety management methodologies (Figure 6) employ different hazard identification methods. Reactive, proactive, and predictive safety management systems all need and use input data, i.e. safety data obtained from different resources. It can also be observed that reactive methodology uses safety data from mandatory occurrence reporting. Proactive methodology uses safety data from mandatory occurrence reporting, voluntary occurrence reporting and data obtained by measuring safety performance (SPIs and SPTs). Predictive methodology uses safety data from mandatory occurrence reporting, voluntary occurrence reporting, data obtained by measuring safety performance (SPIs and SPTs) and data obtained from predictive analyses (forecasts) that extract information from historical and current safety data to predict trends and behaviour patterns of emerging hazards. Safety data obtained from various resources are therefore something that all three methodologies of safety management have in common. Proactive methodology was observed to behave as an upgrade of reactive methodology, and predictive methodology as an upgrade of proactive methodology (Figure 7).

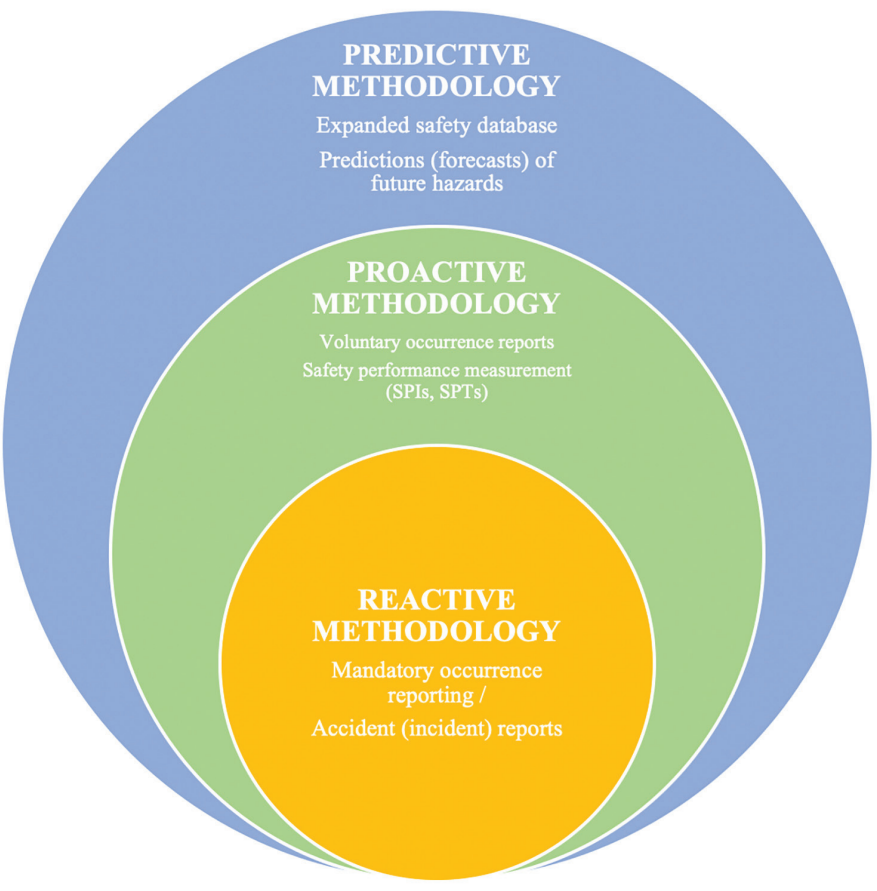

Figure 7.

Correlation between safety management system methodologies.

Source: Author

\section{DEVELOPMENT AND FUTURE APPLICATION OF PREDICTIVE SAFETY MANAGEMENT SYSTEMS}

As described in Chapter 4, there have been attempts to use predictive methods in aviation safety management. All examples show how predictive methods are used in particular safety management segments. The idea is to develop a new model of a predictive safety management system, that would constitute an improvement on the previous reactive and proactive safety management systems, ensure more efficient safety data collection, and facilitate and improve hazard identification, which is the most important step in safety management. While reactive and proactive methodologies identify hazards by collecting safety data from mandatory and voluntary occurrence reports, predictive methodology uses predictive methods to identify potential and possible hazards based on predictive analyses 
(forecasts) that extract information from historical and current safety data to predict trends and behaviour patterns of emerging hazards.

Safety data collected by combining all three methodologies, i.e. the use of a predictive safety management system, can create an extensive and efficient safety database, improve safety performance measurement, and ultimately provide organisations additional infomation about their operations, safety issues and general safety performance.
Predictive safety management system can be applied by any organisation. The focus of this paper was to show how safety management operates, using examples from the aviation industry, but it can be applied in any other transport field, with certain adjustments.

The develpment of a predictive safety management system consists of several phases, as shown in Figure 8.

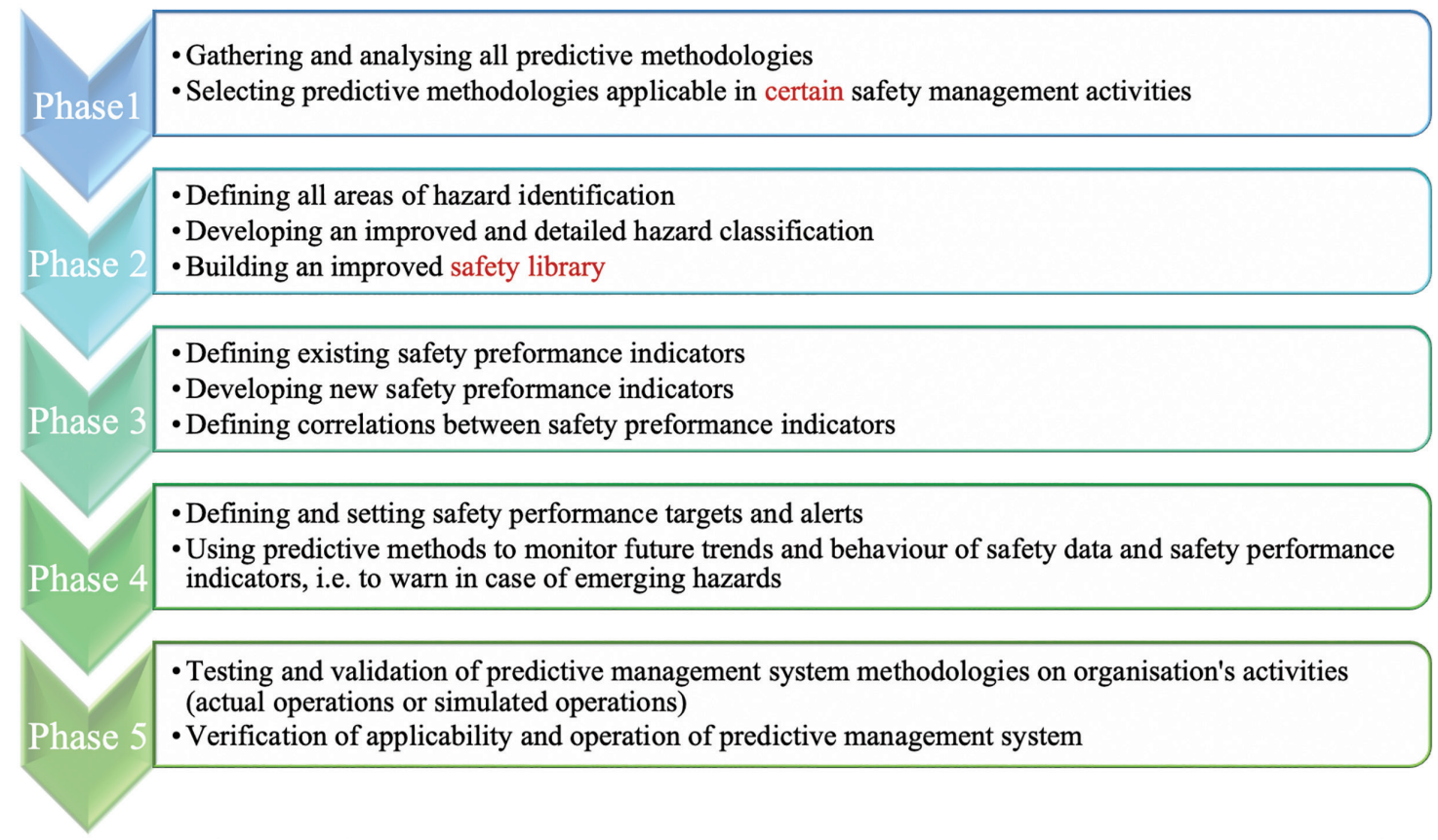

Figure 8 .

Concept of development of a predictive safety management system.

Source: Author

\section{DISCUSSION AND CONCLUSIONS}

Based on research described in this paper, the author has defined the correlations between existing safety management methodologies and proposed the concept for the development of a more advanced safety management system, i.e. a predictive safety management system. The system would include the development of an expanded and well-organised safety database and use predictive (forecasting) methods to identify potential and possible hazards, as well as their trends and behaviour patterns.

Future research will focus on providing an improved breakdown of hazard/ occurrence categories to obtain safety performance indicators for each hazard category vs. timeline and factors influencing them. The categorization of organisation's activities, i.e. factors influencing hazards, will facilitate the breakdown of hazard/ occurrence categories. Definition of hazard/ occurrence categories will help define parameters and indicators that can be monitored and analysed, and ultimately enable us to predict existing or future emerging hazards using predictive safety management system methodology.

Future research will be conducted in several phases. Existing prediction methods applicable to safety management systems will be analysed by means of scientific methods, such as the inductive and deductive method, the analysis and synthesis method, the generalization and specialization method, the proving and refuting method, the classification method, the descriptive method, the compilation method, the comparative method, the statistical method, the mathematical method, the 
modelling method and the experimental method. Based on these and future research results, a predictive safety management system model will be developed.

\section{REFERENCES}

Adjekum, D.K., 2014. Safety Management Systems in aviation operations in the United States: Is the return on investment worth the cost?, Prime Journal of Business Administration and Management (BAM), 4(4), pp. 1442-1450. Available at: http:// www.primejournal.org/BAM/pdf/2014/apr/Daniel\%20pdf.pdf.

African Civil Aviation Commission, 2018. Mechanism for Monitoring Implementation of Safety Systems \& Initiatives, available at: https://www.afcac.org/en/images/ Documentation/mechanism safety.pdf, accessed on: 28 May 2020.

Ancel, E. et al., 2014. Predictive safety analytics: inferring aviation accident shaping factors and causation. Journal of Risk Research, 18(4), pp.428-451. Available at: http://dx.doi.org/10.1080/13669877.2014.896402.

Australian Government, Defence Aviation Safety Authority, 2015. TAREG 4.4.4 Safety Management Systems in Maintenance. Available at: https://www.defence.gov.au/ DASP/Docs/NPRMs/CurrentNPRMs/NPRM01-15.pdf, accessed on: 21 June 2020.

Bangladesh Civil Aviation Authority, 2010. Manual on Aerodrome Safety Management System. Available at: http://www.caab.gov.bd/ano/anoada5.pdf, accessed on: 29 May 2020

Bartulović, D. and Steiner, S., (2020), Liaison Between Proactive and Predictive Methodology of Aviation Safety Management System, Proc. 19th International Conference on Transport Science, Portorož, Slovenia, September 17 - 18, pp. 34-41. Available at: https://icts.sdzp.org/wp/downloads/.

Bartulović, D., 2012. Risk Assessment Methodology in Air Traffic Safety Management System, Master Thesis, Faculty of Transport and Traffic Sciences. Available at: https:// www.bib.irb.hr/720635, accessed on: 29 May 2020.

BHDCA, 2014. Naputak o upravljanju rizicima u sustavu sigurnosti letenja. Available at: http://bhdca.gov.ba/website/dokumenti/Bezbjednost letenja/NAPUTAK rizici hr.pdf, accessed on: 21 June 2020.

Boeing, 2012. Boeing Safety Management System Overview. Available at: http:// www.aviationunion.ru/Files/7\%20En\%20Boeing\%20SMS\%200verview.pdf, accessed on: 29 May 2020

Bohm, F., 2008. Einführung von Safety Management Systemen im Flugbetrieb. Available at: https://www.lba.de/SharedDocs/Downloads/DE/B/B2_Flugbetrieb/ Rundschreiben/94 RS 200816 Praesentation.pdf? blob=publicationFile\&v=1, accessed on: 29 May 2020

Brockwell, P.J. and Davis, R.A., 2016. Introduction to Time Series and Forecasting, 3rd Edition, New York: Springer International Publishing.

Burin, J., 201. Being Predictive in a Reactive World, ISASI Journal, 46(1). Available at: https://www.skybrary.aero/bookshelf/books/3337.pdf, accessed on: 21 June 2020.

Civil Aviation Authority of New Zealand, 2013. Aviation Risk Management, Booklet Four, available at: https://www.aviation.govt.nz/assets/publications/sms-resources/ sms-booklet-4.pdf, accessed on: 29 May 2020.

Čokorilo, , O. and Dell'acqua, G., 2013. Aviation Hazards Identification Using Safety Management System (SMS) Techniques, 16th International Conference on Transport Science (ICTS). Portorož, Slovenia, May 27, pp. 66-73. Available at: https://bib.irb.hr/ datoteka/926003.ICTS_2013_Conference_Proceedings.pdf, accessed on: 21 June 2020.

Čokorilo, O., Ivković, I. \& Kaplanović, S., 2019. Prediction of Exhaust Emission Costs in Air and Road Transportation. Sustainability, 11(17), p.4688. Available at: http://dx.doi.org/10.3390/su11174688.
Croatian Civil Aviation Agency (CCAA), 2016. Implementation of Safety Management Systems (SMS), Air Safety Order ASO-2010-004, Revision 4.

Cusick, S.K., Cortes, A.I. and Rodrigues, C.C., 2017. Commercial Aviation Safety, 6th Edition, New York: McGraw-Hill.

European Union Aviation Safety Agency (EASA), 2011. Commission Regulation (EU) No 1178/2011 laying down technical requirements and administrative procedures related to civil aviation aircrew; Part-ORA.

European Union Aviation Safety Agency (EASA), 2014a. Commission Regulation (EU) No 1321/2014 on the continuing airworthiness of aircraft and aeronautical products, parts and appliances, and on the approval of organisations and personnel involved in these tasks; Part-145 and Part-M.

European Union Aviation Safety Agency (EASA), 2014b. Commission Regulation (EU) No 139/2014 laying down requirements and administrative procedures related to aerodromes, Part-ADR.OR.

European Union Aviation Safety Agency (EASA), 2014c. Regulation (EU) No 376/2014 on the reporting, analysis, and follow-up of occurrences in civil aviation.

European Union Aviation Safety Agency (EASA), 2015. Commission Implementing Regulation (EU) 2015/1018 of 29 June 2015 laying down a list classifying occurrences in civil aviation to be mandatorily reported.

European Union Aviation Safety Agency (EASA), 2012. Commission Regulation (EU) No 965/2012 laying down technical requirements and administrative procedures related to air operations; Part-ORO

Federal Aviation Administration, 2013. FAA's Safety Data Analysis and Sharing System Shows Progress, but More Advanced Capabilities and Inspector Access Remain Limited, available at: https://www.oig.dot.gov/sites/default/files/FAA\%20 ASIAS\%20System\%20Report\%5E12-18-13.pdf, accessed on: 21 June 2020.

Ferguson, M.D. and Nelson, S., 2014. Aviation Safety: A Balanced Industry Approach, 1st Edition, New York: Delmar.

GAIN Working Group B, Analytical Methods and Tolls, 2003. Guide to Methods \& Tools for Airline Flight Safety Analysis, available at: https://flightsafety.org/wpcontent/uploads/2016/09/methods_tools_safety_analysis.pdf, accessed on: 28 May 2020.

Hsiao, Y.-L. et al., 2013. Predictive models of safety based on audit findings: Part 1: Model development and reliability. Applied Ergonomics, 44(2), pp.261-273. Available at:

http://dx.doi.org/10.1016/j.apergo.2012.07.010

Hsiao, Y.-L. et al., 2013. Predictive models of safety based on audit findings: Part 2 : Measurement of model validity. Applied Ergonomics, 44(4), pp.659-667. Available at: http://dx.doi.org/10.1016/j.apergo.2013.01.003.

ICAO, IATA, IFALPA, 2015. Fatigue Management Guide for Airline Operators, Second Edition. Available at: https://www.icao.int/safety/fatiguemanagement/FRMS\%20 Tools/FMG\%20for\%20Airline\%200perators\%202nd\%20Ed\%20(Final)\%20EN.pdf, accessed on: 28 May 2020

International Civil Aviation Organisation (ICAO), 2016. Annex 19 to the Convention on International Civil Aviation: Safety Management, Second Edition.

International Civil Aviation Organisation (ICAO), 2018. Safety Management Manual (SMM), Doc 9859, Fourth Edition.

International Civil Aviation Organization (ICAO), 2013. Global Aviation Safety Plan, Doc 10004, 2014-2016 Edition.

International Civil Aviation Organization (ICAO), 2019. Global Aviation Safety Plan, Doc 10004, 2020-2022 Edition. 
International Civil Aviation Organization, (2011), Safety Management Principles. Available at: https://www.icao.int/APAC/Meetings/2011sip/WP.19\%20-\%20Safety. pdf, accessed on: 29 May 2020.

International Civil Aviation Organization, (2013), State of Global Aviation Safety. Available at: https://www.icao.int/safety/State\%20of\%20Global\%20Aviation\%20 Safety/ICAO SGAS book EN SEPT2013 final web.pdf, accessed on: 21 June 2020.

ITF Roundtable Reports. Available at: http://dx.doi.org/10.1787/25198785.

Jakovljević, I. et al., 2017, Aircraft Departure Control Systems-Hidden Safety Risks, International Journal for Traffic and Transport Engineering (IJTTE), 7(3), pp. 298-311. Available at:

https://doi.org/10.7708/ijtte.2017.7(3).02.

Khoshkhoo, R., 2017. Adaptation of Line Operations Safety Audit (LOSA) to Dispatch Operations (DOSA). Journal of Airline and Airport Management, 7(2), p.126. Available at:

http://dx.doi.org/10.3926/jairm.112.

Liou, J.J.H., Yen, L. \& Tzeng, G.-H., 2008. Building an effective safety management system for airlines. Journal of Air Transport Management, 14(1), pp.20-26. Available at: http://dx.doi.org/10.1016/j.jairtraman.2007.10.002.

Luxhøj, J.T., 2013. Predictive Safety Analytics for Complex Aerospace Systems. Procedia Computer Science, 20, pp.331-336. Available at: http://dx.doi.org/10.1016/j.procs.2013.09.281.

NavBlue, Flight Data Analysis, 2020. Available at: https://www.navblue.aero/ product/flight-data-analysis-suite/, accessed on: 19 August 2020.

Ordinance on the implementation of Regulation (EU) 376/2014, 2015. Official Gazette, 2015(107), Official Gazette, 2016(92), Official Gazette, 2019(28).

Oster, C.V., Strong, J.S. \& Zorn, C.K., 2013. Analyzing aviation safety: Problems, challenges, opportunities. Research in Transportation Economics, 43(1), pp.148164. Available at:

http://dx.doi.org/10.1016/j.retrec.2012.12.001.
Panagopoulos, I., 2011. Flight Safety in Combat Training: A revised pilot's error framework for EU Air Forces, 5th Biennial Hellenic Observatory PhD Symposium, UK, London, June 2 - 3, pp. 1-8. Available at: https://pdfs. semanticscholar.org/6f2e/0a4546af6538f60933dc0c5828e4dcd88dc2.pdf? ga $=2.19692400 .993315240 .1599139638-339680789.1599139638$, accessed on: 29 May 2020.

Pisanich, G.M. and Corker, K., 1995. A Predictive Model of Flight Crew Performance in Automated Air Traffic Control and Flight Management Operations, Ohio State 8th International Symposium on Aviation Psychology, USA, Columbus, Ohio, April 24 -27 .

Roelen, A.L.C. et al., 2016. Risk models and accident scenarios in the total aviation system. Available at: https://ascos-project.eu/downloads/ascos paper roelen.pdf, accessed on: 28 May 2020.

Steiner, S., 1998. Elements of Air Traffic Safety - Elementi sigurnosti zračnog prometa. $1^{\text {st }}$ Edition, Zagreb: Faculty of Transport and Traffic Sciences.

Stanton, N.A. et al., 2009. Predicting pilot error: Testing a new methodology and a multi-methods and analysts approach. Applied Ergonomics, 40(3), pp.464-471. Available at:

http://dx.doi.org/10.1016/j.apergo.2008.10.005.

State Safety Programme, 2015. Official Gazette, 2015(141).

Stolzer, A.J. and Goglia, J.J., 2015. Safety Management Systems in Aviation, $2^{\text {nd }}$ Edition, Farnham: Ashgate.

The Airbus Safety Magazine: Safety First, 2014. Flight Data Analysis (FDA), a Predictive Tool for Safety Management System (SMS), 17. Available at: https://safetyfirst.airbus. com/app/themes/mh newsdesk/pdf.php?p=25663, accessed on: 29 May 2020

Velazquez. J. and Bier, N., 2015. SMS and CRM: Parallels and Opposites in their Evolution, Journal of Aviation/Aerospace Education \& Research, 24(2), pp. 55-78. Available at:

https://doi.org/10.15394/jaaer.2015.1616. 